\title{
The Logic of Factor Analysis
}

\author{
-Discussions from the \\ Methodological Point of View-
}

\author{
by TAROW INDOW \\ Keio University, Tokyo
}

Nobody will deny that factor analysis is essentially a statistical method. Presumably it may be said to belong to multivariate analysis. Nevertheless, strange to say, it has escaped until recently the attention it deserves among mathematical statisticians $[4,10,12,13,15]$. A few mathematicians, for example Hotelling [11], have taken an interest in it. However, the main development of this technique, at least in its early stage, is entirely due to psychologists, i. e., Spearman [17], Thurstone [19], and Thomson [18] etc. Its history seems, thus, very suggestive of that the germ of the birth and development of factor analysis consists in a peculiarity of psychological measurements.

In so called psychological tests, a mental trait of an examinee is commonly measured in terms of a test score which is obtainable from the examinee's responses to a set of test items, but the true target of test is always beyond our observation and control. The mental trait is a hypothetical variable postulated as underlying the responses. In a word, measurements in psychology may be said indirect in nature. The operation is doomed to be radically different from applying a measure directly to an object under a fixed gaze and a psychologist has to grope in the dark for something solid to which he will give the name of so and so mental trait. No wonder, psychologists have been in pursuit of a device to help them in their groping and, as will be stated later, the need seems to have given birth to factor analysis. 


\section{Brief Description of Factor Analysis}

Let us suppose that an experimental population of $N$ individuals has been given a set of $n$ tests (test battery), the resulting tabulation of scores will be $n \times N$ matrix, whose elements are the scores $x_{i j}$, where the subscript $i$ refers to the examinee and $j$ refers to the test. In factor analysis a group of tests, not a single test, are always taken into consideration at a time. As a matter of course, innumerable ways are conceivable to quantify a given set of responses of an examinee $i$ to the items in a test $j$ as $x_{i j}$. A question will be raised, then, as to what measure should be chosen in order to establish an appropriate relationship between the test score and the ability underlying the responses. This is the problem of scaling $[8,14,22]$ which will not be discussed in the present article. If we take $x_{i j}$ for granted in whatever measure it may be given, then the problem at hand is to account for the scores, as they stand, in terms of fundamental factors of as small number as possible with the hope or conviction that the domain of mental activities being covered by the test battery is not so chaotic as it looks and structured as follows :

$$
x_{i j}=\sum_{s=1}^{m} b_{j s} F_{s i}+u_{1 j} S_{j i}+u_{2 j} U_{j i}
$$

where $F_{s}$ represents a mental trait operating in the responses to two or more tests in the battery (common factor), $S_{j}$ represents a trait which is involved only in a test $j$ (specific factor), and $U_{j}$ is the error term. The subscript $i$ in these factors is to indicate the magnitude of the respective factor possessed by an examinee. It is convenient to define each of $x, F, S$, and $U$ in standard form; the origin at the mean and the standard deviation as the unit. Obviously no generality is lost by this. The coefficients in (1) are called loadings and represent contributions of the corresponding factors to variations in the scores. For instance, the degree of dependence of score of a test $j$ upon magnitude 
of a common factor $s$ will be shown by $b_{j s}$. By definition $S_{j}$ and $U_{j}$ are uncorrelated with $F_{s}$ and also among themselves. Then, by use of matrix notation, it can be easily shown:

$$
R=B R_{F} B^{\prime}
$$

where

$$
R=\left(\begin{array}{cccc}
h_{1}{ }^{2} r_{12} \cdots \cdots \cdot r_{1 n} \\
r_{21} h_{2}{ }^{2} \cdots \cdots \cdot r_{2 n} \\
\vdots & \vdots & \ddots & \vdots \\
\vdots & \vdots & \ddots & \vdots \\
r_{n 1} r_{n 2} & \cdots \cdots h_{n}{ }^{2}
\end{array}\right)
$$

which is called the correlation matrix, whose off-diagonal elements are the product-moment correlation coefficients between all possible pairs of the test scores, $B$ is the common factor loading matrix consisting of $b_{j s}$, and $R_{F}$ gives the correlations among the common factors. A matrix followed by a prime, like $B^{\prime}$, is hereafter to be understood as the transpose of the original matrix. The matrix $R$ is calculable from the score matrix $X$ in the form:

$$
R=\frac{1}{N} X X^{\prime}
$$

except for the elements in the main diagonal which are to be defined as

$$
h_{j}{ }^{2}=\sum_{s} \sum_{t} b_{j s} b_{j t} r\left(F_{s}, F_{t}\right)=1-\left(u_{1 j}{ }^{2}+u_{2 j}{ }^{2}\right)
$$

and are called communalities. The term $r\left(F_{s}, F_{t}\right)$ is an element in the matrix $R_{F}$.

Factor analysis can be considered as involving two basic steps: the factoring and the rotation of axes, i. e., the search for the factors psychologically meaningful. In the former, an attempt is made to account for the score matrix $X$ in terms of an arbitrary set of uncorrelated factors of the smallest possible number, $m$. Mathematically this is equivalent to determine the rank of $R$. Let the uncorrelated factors be denoted by $F^{\prime} s$ and the loadings in this case by $a_{j s}$, then the first term of the right member of (1) becomes $\sum_{s} a_{j s} F^{\prime}{ }_{s i}$, and instead of (2), as $R_{F}$ becomes an identity matrix, 


$$
R=A A^{\prime}
$$

where $A$ consists of $a_{f s}$. The factoring is carried ordinarily as follows : the column vector $\left(a_{j s}\right)$ is included one by one into the matrix $A$ as one of its columns until the residuals in the matrix $\left(R-A A^{\prime}\right)$ are judged negligible. Mathematically stated, the equation (4) does not hold unless $m$ characteristic roots of $R$ are positive and the remaining $n-m$ characteristic roots vanish. If $R$ can be resolved into $A$ in the form of (4), a test $j$ may be represented by a point $\left(a_{j 1}, a_{j 2}, \cdots, a_{j m}\right)$ in the $m$-dimensional Euclidean space. This is a statistical condensation of $n$ kinds of the observed variables into $m$ arbitrary parameters, which may be considered to be successful if $m$ is much smaller than $n$. As mentioned above, the left member of (3) lacks in its main diagonal, the communalities. In theory, however, the minimum rank possible of $R$ can be completely determined by the structure inherent in its off-diagonal elements $r_{j k}$. For example, it is impossible in the matrix of $n=4$ to assume $m=1$ unless every two-rowed minors of $R$ such as

$$
\left|\begin{array}{ll}
r_{13} & r_{14} \\
r_{23} & r_{24}
\end{array}\right|
$$

vanishes. By virture of these relationships in the empirical data, it becomes possible to consider, by a suitable choice of $h_{j}{ }^{2}$, that $R$ is of rank one in this case. Some of the minors involve $h_{j}{ }^{2}$ :

$$
\left|\begin{array}{ll}
h_{1}^{2} & r_{13} \\
r_{21} & r_{23}
\end{array}\right|,\left|\begin{array}{ll}
h_{1}^{2} & r_{14} \\
r_{21} & r_{24}
\end{array}\right|,\left|\begin{array}{ll}
h_{1}^{2} & r_{14} \\
r_{31} & r_{34}
\end{array}\right|
$$

Should the rank of $R$ be reduced to the minimum, hence, it is the value

$$
h_{1}^{2}=\frac{r_{12} r_{13}}{r_{23}}=\frac{r_{12} r_{14}}{r_{24}}=\frac{r_{13} r_{14}}{r_{34}}
$$

that should be put into the analysis as the first elements of the main diagonal of $R$ in (4). In accordance with the relationships standing among the observed variables, the communalities thus estimated will provide us with the minimum number of factors necessary to account for the facts of observation. In this way the determination of the rank of $R$ is made according to the principle of parsimony with the result 
that the configuration of $n$ points representing the tests is contained in the space of the smallest possible dimensionality.

The next step in factor analysis is to find a solution for the problem where to locate, in the configuration of test points, $m$ coordinate axes which should depict all the essential features of the mental traits underlying the domain at issue. The factors obtained in the process of factoring, $F^{\prime}{ }_{s}$, are only for the sake of convenience to give a concrete form to the configuration and to visualize it. On the contrary, each factor in (2) must be psychologically meaningful in the sense that $F_{s i}$ reveals a trait $s$ in an individual $i$. Again, one of the principles applied in this connection will be that of parsimony.

One of the turning-points in the solution is, according to Thurstone, the concept of simple structure. In factoria1 studies investigators proceed on the assumption that the domain under discussion is structured somewhat, that underlying factors do not all participate equally in every mental activity belonging to the domain in so far as they are distinguishable from each other in function. In other words, a number of loadings should vanish in each row of the matrix $B$ in which all the elements are $m$ in total. If such a matrix $B$ is discovered obtainable by an appropriate transformation from the matrix $A$ that reveals a pattern of non-zero loadings of a relatively small number being relieved in the remaining loadings vanishing, and besides, if the pattern of significant loadings has such a property to make explicit the nature and content of the newly established factors, the combination of the two matrices $B$ and $F$ is called simple structure and is conceived as representing the structure inherent in the domain. The common factors $F_{s}$ thus arrived at are in general not independent to each other and the intercorrelations among them are given in the matrix $R_{F}$. Geometrically interpreted, the matrix $B$ corresponds to an appropriate set of oblique coordinate axes in the $m$-dimensional space containing the configuration of test points and the transformation

$$
B=A T
$$


is essentially rotation of the axes. The matrix $R_{F}$ will be obtained as follows :

$$
R_{F}=\left(T^{-1}\right)\left(T^{-1}\right)^{\prime}
$$

Someone refers to simple structure as the simple idea of finding the smallest number of parameters for describing each test. Then the result is obviously the most parsimonious from the numerical viewpoint. Should this criterion that is solely based on the numerical parsimony be adopted, simple structure will be arrived at analytically. A number of analytical solution for simple stucture have been proposed by several authors $[5,16,20,24$,$] , and these methods will release investigators$ from trial-and-error in the intuitive-graphical solution. As a matter of fact, it has been repeatedly shown that correspondence exists between the result analytically obtained and the one reached intuitively by graphical rotation of axes. Discrepancies, if they occur, may be due to weights assigned by investigators to points (6). In rotating axes, investigators may attach more or less importance to some points than to others, which evidently comes from another criterion besides the numerical parsimony. Sometimes the pattern of non-zero loadings may be conceivable that gives us clearer understanding as to the nature and content of factors, though it is less parsimonious from the numerical viewpoint. If the suspicion is to be dispelled that a simple structure and interpretation of factors reached by an investigator is not free from his personal bias, with the given matrix $A$ the intuitive-graphical rotation of axes should be tried independently by several investigators in order to see whether an unanimous solution is reached, which has been actually done in some cases.

In empirical studies, factor analysis has not infrequently been applied in various kinds of domain with the result of beautiful simple structure. For example, in the field of intelligence tests factors have been repeatedly identified such as verbal, spatial, number, memory, mental speed, induction and deduction etc. [7, 21]. It is in terms of these functional unities that fundamental differences in intellectual endowment among 
men are to be interpreted. The list will not be exhaustive until the entire domain to be called intellectual activities is completely explored. Nevertheless, it is true that at least a part of the hidden structure has come to light through factor analytic studies, that individual differences in intellectual activities, numberless as directly observable variables, seem to be reduced to fundamental differences in the basic mental abilities which are certainly limited in number even when exhaustively enumerated.

Hierarchy of Analytical Procedures in

Empirical Sciences and Factor Analysis

As a part of the logic of factor analysis, the role of individual differences is worth mentioning. Factor analysis makes use of the existence of individual differences as an avenue of approach to the underlying factors. By virtue of it, it becomes possible to give an operational definition to a mental ability which itself is not directly observable and to distinguish an ability from each other as a functional unity. Discussions concerning the role of individual differences in factor analysis will be helpful to clarify the peculiar circumstances in psychological measurement that have spurred psychologists on to develop an appropriate statistical technique of their own.

Of an equation for a test $j$ in (1), the left member $x_{j}$ will be ordinarily called a dependent variable and the first $m$ terms $F_{s}$ in the right member independent variables if the last two therein are regarded en bloc as the error terms. If all the independent variables at issue are under control of an investigator, he will change at will the independent variables and observe the concomitant change in the dependent variables. This is an orthodox procedure in experimental sciences to establish a functional relationship between the dependent and independent variables. Should the relationship be discovered not to be linear as in (1), the investigator may have no difficulty to describe it with a 
more complex equation provided that the experimental data available are with sufficient accuracy for that purpose. As it were, everything may be said to be in broad daylight and carried out under a fixed gaze of investigators in the orthodox approach. Needless to say, psychologists will not hesistate in taking the orthodox approach if possible, simply because that belongs to the first and top stage of the hierarchy conceivable among analytical procedures in empirical sciences.

On the other hand, an equation in (1) may be considered as a multiple regression equation of $x_{j}$ upon a set of $m$ independent variables $F_{s}$. Multiple regression analysis will be applied to the situation in which the independent variables are beyond experimenter's control but still measurable in the quantitative term. Hence, multiple regression analysis may be said to belong to the second stage of the hierarchy. Therein independent variables $F_{s}$ as well as the dependent variable $x_{j}$ are the given data and what is to be determined by analysis is a set of coefficients $b_{j s}$. Instead of making measurement, to give the dependent variable, of objects in various prescribed states with respect to the independent variables, the experimenter obtains from each object the informations concerning not only with $x_{j}$ but also with $F_{s}$ and in virture of variations among the objects existing by nature with respect to $F_{s}$ a functional relationship may be established between $x_{j}$ and $F_{s}$ in the same way as by the orthodox procedure. If it becomes apparent that the linear relationship such as in (1) is not appropriate, it may not be impossible in theory to conceive of a more complex equation to fit the data. It is evident that psychologists often have to deal with factors beyond their control. For instance, when an assumption is made that among other factors memory is taking the leading role in so and so activity, it is entirely out of question to give variation at will to the ability of memory of an individual in order to observe the concomitant change in his activity under discussion or it may be tedious to select several samples of people each of which is in a prescribed state concerning the ability of memory in order to measure differences in 
the actitvity among the samples. Hence, by considering $N$ individuals as a whole the investigator will make use of individual differences which certainly exist by nature in the ability of memory to give variation to the dependent variable and to the independent variables also. It is to be noted, however, that a prerequisite to the multiple regression analysis mentioned above is the assumption that there exists a psychological entity such as the ability of memory and that it can be measured independently of any other factor. If this assumption is not to be taken for granted, we are certainly in the third stage of the hierarchy.

Not infrequently psychologists encounter a situation where they have no exact notion of the factors involved or where the anticipated factor is not only beyond their control but also not measurable independently of any of other factors. Factor analysis has been developed as a method applicable to such a situation. Multiple regression analysis can be properly applied to a situation where the independent variables are observable yet out of the investigator's control whereas factor analysis is only for cases where the variables formally corresponding to the indipendent variables are not observable and consequently out of control. In the equations (1), the input to analysis remains a set of $n$ kinds of variables $x_{j}$ only and all the others belong to the output, not only the nature of $F_{s}$ to be considered and the corresponding coefficients $b_{j s}$ but even the number of $F_{s}$, i. e., $m$, are not known until the analysis is completed. Ordinarily even the composer of a test is not quite certain about what is involved in it. The score of a test composed for the purpose of measuring the ability of memory, for instance, might be also functions of additional factors, or there might be no independent functional unity such as the ability of memory. Factor analysis will be useful to clear a path for making explicit that which is involved in each test by means of putting several tests together into analysis. In factor analysis, as in multiple regression analysis, a group of examinees are taken into consideration as a whole in order to make use of their 
individual differences and, besides, a number of tests have to be analyzed simultaneously so that the tests give form, hand in hand, to the functional unities involved in common in two or more tests. However, restrictions will be imposed inevitably upon its successful application that the observable $x_{j}$ should be expressed as a linear combination of the hidden factors $F_{s}$, that a set of the factor loadings $b_{j s}$ should hold good in common to all the members in the group. Individual differences are assumed to be explainable entirely in terms of $F_{s}$ and as to $b_{j s}$ no variation is allowed from individual to individual. In a word, the examinees must be homogeneous in the mental structure. In multiple regression analysis the assumption is made about the latter only, since the former is open to experimental test and, if necessary, the linear equation can be replaced by a more appropriate equation as mentioned before. On the other hand, if the model, i. e., both of linearity and homogeneity, does not fit the structure inherent in the domain, the application of factor analysis will result in nothing. So to speak, applying factor analysis may be said to be something like gambling. Even when the model holds, the investigator can not be too cautious in ascertaining that the analysis fits the mark and the results reveals the real features of the structure because the result of factor analysis is, as it were, something groped in the dark. Whenever a factorial study is carried out, we obtain a result in any case, which might or might not be the true description of hidden factors, however. Certainly it may happen that fictitious factors are artificially produced in the process of analysis. Hence the problem is to make a distinction in the result of factor analysis between a true discovery of the structure and an artifact.

\section{Factoriol Invariance as a Fundamental Criterion for the Operational Definition of Mental Traits}

Having arrived at the simple structure in an analysis may not be sufficient to ascertain that the analysis hits the mark. Should the 
result be not an artifact, the simple structure remains invariant when the analysis is repeated with a slight modification in the input. Demonstrating invariance seems to be the only one method available to distinguish a true discovery of hidden factors from an artificial result produced in the process of analysis. To repeat the analysis under exactly the same conditions, i. e., with the same test battery and with the equivalent sample of examinees from the same population, will provide us with little information in this respect. It is a matter of reliability but not of invariance. One of the means to make the modification is to move a test $j$ in a battery to another battery involving the same common factors. If essentially the same factorial composition $\left(b_{j 1}, b_{j 2}, \cdots, b_{j m}\right)$ is obtained in the two analyses, with the first and second batteries, the result will be satisfactory even to most critical investigators. Mathematically no difficulty is involved with respect to the invariance of this kind*. A practical difficulty may consist in setting up the second battery which should involve as many the same common factors with the original battery as possible. Even when a few of the common factors in the original battery are lacking in the second, however, the invariance will still be obtained with the remaining ones.

A modification in sampling of examinees offers, however, a problem which is mathematically of great interest. It is well known that the value of correlation coefficient $r_{j k}$ is not independent of the ranges of $x_{j}$ and $x_{k}$. Hence, if the same battery of tests is administered to two groups of examinees differing in range, the two correlation matrices cannot be the same. What occurs, then, with the final results of the two factor analyses which start from the different correlation matrices? Let us suppose that examinees are heterogeneous to the fullest extent in the first analysis and a simple structure has been obtained, that examinees in the second analysis are more or less restricted in the

* Recently Tucker discussed a problem of mathematical interest in this line [25]. 
range of several test scores by a selection, then, is it possible mathematically to expect invariance between the results of these analyses? If it becomes theoretically clear that simple structure does not remain invariant under selection of examinees, it will be fatal to factor analysis as a tool in empirical sciences [9], because in its empirical application investigators cannot entirely steer clear of the influence of selection. For convenience's sake only, students in a school are often used as subjects in an analysis. Evidently they are more or less restricted with respect to the ranges in age, abilities etc. Theories concerning the effect of selection of examinees upon factorial results has been studied by Thurstone [19], Thomson [18], Tucker [23] and Ahmavaara [1].

In order to study the influence of selection, it is convenient to divide the observable variables into two groups, namely, tests which are directly involved in the selection and tests which are not directly involved. Hereafter, the former, the selection tests, will be denoted by the subscript $j$ and the others by the subscript $k$. Let the matrix of correlations and variances before selection be represented by $\mathfrak{R}$ and that after selection by $\mathfrak{R}^{*}$. The letter with asterisk should be understood hereafter as designating the counterpart after selection. Namely, by selection $\mathfrak{R}$ changes to $\mathfrak{R} *$ as follows,

$$
\left(\begin{array}{ll}
\Re_{j j} & \Re_{j k} \\
\Re_{k j} & \Re_{k k}
\end{array}\right) \stackrel{\text { selection }}{\longrightarrow}\left(\begin{array}{ll}
\Re_{j j}^{*} & \Re_{j k}^{*} \\
\Re_{k j}^{*} & \Re_{k k} *
\end{array}\right)
$$

where $j=1,2, \cdots, l, k=l+1, l+2, \cdots, n$ and the diagonal elements are variances of $x_{j}$ or $x_{k}$, not communalities, in both matrices. Then, it has been shown by Aitken,

$$
\begin{gathered}
\Re_{j k} *=\Re_{j j} * \Re_{j j}-1 \Re_{j k}=\left(\Re^{*}{ }_{k j}\right)^{\prime} \\
\Re_{k k} *=\Re_{k k}-\Re_{k j}\left(\Re_{j j}{ }^{-1}-\Re_{j j}{ }^{-1} \Re_{j j} * \Re_{j j}{ }^{-1}\right) \Re_{j k}
\end{gathered}
$$

under the influence of the selection $\Re_{j j} \rightarrow \Re_{j j} *$. From these formula, Ahmavaara has shown that selection, as regards its effects on correlations and variances, is equivalent to a linear transformation in the selection space which contains $l$ linearly independent vectors repre- 
senting the selection tests $x_{j}$ in their full lengths $(=1)$.

Let us consider the loading matrix in the selection space, $A_{0}$, and the linear transformation therein $L_{0}$, then

$$
A_{0} L_{0}=\left(\begin{array}{ll}
A_{j} & U_{j} \\
A_{k} & 0
\end{array}\right)\left(\begin{array}{l}
L_{c} \\
L_{u}
\end{array}\right)=\left(\begin{array}{l}
A_{j} L_{c}+U_{j} L_{u} \\
A_{k} L_{c}
\end{array}\right)
$$

where $U_{j}=\left(u_{j 1}, u_{j 2}\right)$ and $L_{c}$ refers to the transformation in the common factor sub-space of the selection space and $L_{u}$ refers to the transformation in the remaining part of the selection space. However, factor analysis after selection will start from the correlation matrix $R^{*}$, and analyze it, as the first step, in the form corresponding to (4), which can be written as

$$
R^{*}=D\left(A_{0} L_{0}\right)\left(A_{0} L_{0}\right)^{\prime} D=\left(D A_{0} L_{0}\right)\left(D A_{0} L_{0}\right)^{\prime}=A^{*}\left(A^{*}\right)^{\prime}
$$

where $D$ is a diagonal matrix to express the test scores after selection in the standard form, $i$. e., to change the diagonals from variances to communalities. It is easily shown that $A^{*}$ is of rank $(m+l)$ where $m$ is the rank of $A$. However, the part of $R^{*}$ corresponding to $R_{k k}$ is given by

$$
R_{k k^{*}}=\left(D_{k} A_{k} L_{c}\right)\left(D_{k} A_{k} L_{c}\right)^{\prime}=A_{k} *\left(A_{k}^{*}\right)^{\prime}
$$

and $A_{k}{ }^{*}$ is of rank $m$. Ahmavaara summarized what described above in the theorem: the number of common factors to be involved is not increased by selection except in the selection tests, in which there appear at most as many additional factors as there are selection tests.

The final result of factor analysis is the loading matrix representing psychologically meaningful factors that is derived, in the case of selection, from $A^{*}$ in the form corresponding to (5)

$$
B^{*}=A^{*} T^{*}
$$

and, as $A^{*}=B^{*}\left(T^{*}\right)^{-1}$, by analogy of (11)

$$
\left(T^{*}\right)^{-1}=D_{t} T^{-1} L_{c}
$$

where $L_{o}$ is replaced by $L_{c}$ because only the common factor subspace in the selection space is at issue and $D_{t}$ is a diagonal matrix normalizing the rows of $\left(T^{-1} L_{c}\right)$.

As regards $B^{*}$, Ahmavaara has shown a kind of invariance in the 
non-selection tests. By means of (5), (12), (14),

$$
B_{k} *=A_{k} * T^{*}=D_{k} A_{k} L_{c} L_{c}{ }^{-1} T D_{t}=D_{k} A_{k} T D_{t}=D_{k} B_{k} D_{t}^{-1}
$$

were $B_{k}{ }^{*}$ is the part of $B^{*}$ corresponding to the non-selection tests. Since $D_{k}$ and $D_{t}^{-1}$ are diagonal matrices, the elements vanishing in $B_{k}$, $b_{k s}=0$, still appear as $b_{k s} *=0$ so that the part of the simple structure corresponding to non-selection tests remains invariant as a pattern from $B_{k}$ to $B_{k} *$ through the selection. The invariance does not hold numerically however. Of importance in factorial result may be a pattern revealed by the loadings but not the value itself of each loading.

Theoretically considered, however, even the invariance as a pattern does not hold as regards $B_{j}$, the part of $B$ consisting of the selection tests, besides, as shown before, there appear additional factors. None the less, the conclusions in theory leave it an open question whether or not selection, except for extreme cases, exert in practice so destructive an influence upon factorial result as to mislead investigators with respect to the number and nature of factors. As mentioned before, factoring is stopped when the residuals in the matrix $\left(R-A A^{\prime}\right)$ becomes negligible in magnitude and, in fact, it is a sudden drop in the values of characteristic roots of $R$ and not almost complete vanishing of the residuals that gives investigators the conviction that significant factors have been exhausted. If no abrupt decrease is observed of the values of chracteristic roots, the analysis should be discarded as a failure because there is no hope to reach an objective result. From the practical point of view, therefore, it may not be too optimistic to assume that in most cases of moderate selection the additional factors are not discernible from the error terms in factoring. And if no additional factor creeps in, the deformation of factorial result due to the selection may remain, if any, to a degree that does not obscure the insight of investigators into the real structure of domain when rotating axes.

Since factor analysis is a method to grope in the dark for something directly invisible, the investigators may be said, in a sense, to overtask themselves and the only one method available to protect them against 
the danger of grasping something wrong being misled in the process of analysis may be to ascertain invariance of the result by repeating the analysis with different batteries or with different groups of examinees. Satisfactory invariance has been demonstrated in some cases [e. g. 2, 3].

As mentioned in the beginning of this article, measurements in psychology are in general indirect in nature, that is, investigators cannot observe directly a mental trait and have no operation to isolate it. Hence, in order to give a concrete form to something underlying mental activities involved in a test, it becomes inevitable to invoke a method such as factor analysis. And also, in order to make the result of factor analysis "objective", it seems necessary to rely upon a criterion such as invariance. In a word, therefore, the invariance as discussed so far may be said a fundamental criterion available for psychologists to give the operational definition to any mental trait. The process yielding the invariant output irrespective of modifications in the input of analysis may be admitted as the subject of psychological studies, in the same way as the property of figure remaining invariant in the group of transformations constitutes the subject of geometry according to the Erlangen program.

\section{Reference}

[1] Ahmavaara, $Y$. The mathematical theory of factorial invariance under selection. Psychometrika, 1954, 19, 27-38.

[2] Ahmavaara, Y. On the unified factor theory of mind.. Stomalaisen Tiedeakatemian Toimituksia. Annales Akademie Scientiarum Fennice, Helsinki 1957.

[3] Ahmavaara, Y., \& Markkanen, T. The unified factor model. Its position in psychometric theory and application to sociological alcohol study. The Finnish Foundation for Alcohol Studies. Vol. 7. 1958.

[4] Anderson, T. W., \& Rubin, H. Statistical inference in factor analysis. In Neyman, J. (Ed.), Proceedings of the third Berkeley symposium on mathematical statistics and probability. The University of California Press, 1956, 111-150.

[5] Carrol, J. B. An analytical solution for approximating simple structure 
in factor analysis. Psychometrika, 1953, 18, 23-38.

[6] Fergurson, G. A. The concept of parsimony in factor analysis. Psychometrika, 1954, 19, 281-290.

[7] French, J. W. The description of aptitude and achievement tests in terms of rotated factors. Psychometric Monog., 1951, No. 5.

[8] Guilford, J. P. Psychometric methods. McGraw-Hill 1954 (second edition.)

[9] Henrysson, S. Applicability of factor analysis in the behavioral sciences. Acta Universititatis Stockholmensis Stockholm Studies in Educational Psychology 1. 1957 Almqvist \& Wilsell.

[10] Higman, B. Applied group-theoretic matrix methods. 1955, Oxford University Press.

[11] Hotelling, H. Analysis of a complex of statistical variables into principal components. J. educ. Psychol., 1933, 24, 417-441, 498-520.

[12] Kenda11, M. G., A course in multivariate analysis. Charles Griffin, 1957.

[13] Kenda11, M. G., \& Smith, B. B., Factor analysis. Jour. of the Royal Statisttical Society, Series B (methodologica1) 1950, 12, 60-94.

[14] Lord, F. A theory of test scores. Psychometric Monog. 1952.No. 7.

[15] Rao, C. R. Estimation and tests of significance in factor analysis. Psychometrika, 1955, 20, 93-111.

[16] Sokal, R. R. Thurstone's analytical method for simple structure and a mass modification thereof. Psychometrika, 1958, 23, 237-257.

[17] Spearman, C. The abilities of man. MacMillan, 1927.

[18] Thomson, G. The factorial analysis of Human ability. University of London Press. (4th ed.) 1950.

[19] Thurstone, L. L. Multiple-Factor Analysis. A development and expansion of the Vectors of Mind. The University of Chicago Press. 1947.

[20] Thurstone, L. L. An analytical method for simple structure. Psychometrika, 1954, 19, 173-194.

[21] Thurstone, L. L. \& Thurstone, T. G. Factorial studies on intelligence. Psychometric Monog. 1941, No. 2.

[22] Torgerson, W. S. Theory and methods of scaling. Wiley, 1958.

[23] Tucker, L. R. The role of correlated factors in factor analysis. Psychometrika, 1940, 5, 141-152.

[24] Tucker, L. R. The objective definition of simple structure in linear factor analysis. Psychometrika, 1955, 20, 209-225.

[25] Tucker, L. R. An inter-battery method of factor analysis. Psychometrika, 1958, 23, $111-136$. 